\title{
Genetic Control of Circuit Function: Vsx 1 and Irx 5 Transcription Factors Regulate Contrast Adaptation in the Mouse Retina
}

\author{
Daniel Kerschensteiner, ${ }^{1,2}$ Haiquan Liu, ${ }^{3}$ Chi Wa Cheng, ${ }^{4}$ Jay Demas, ${ }^{5}$ Shuk Han Cheng, ${ }^{4}$ Chi-chung Hui, ${ }^{6,7}$ \\ Robert L. Chow, ${ }^{3}$ and Rachel 0. L. Wong ${ }^{1,2}$ \\ ${ }^{1}$ Department of Anatomy and Neurobiology, Washington University School of Medicine, St. Louis, Missouri 63110, ${ }^{2}$ Department of Biological Structure, \\ University of Washington, Seattle, Washington 98195, ${ }^{3}$ Department of Biology, University of Victoria, Victoria, British Columbia, Canada V8W 3N5, \\ ${ }^{4}$ Department of Biology and Chemistry, City University of Hong Kong, Kowloon, Hong Kong, China, ${ }^{5}$ Cold Spring Harbor Laboratory, Cold Spring Harbor, \\ New York 11724, and ${ }^{6}$ Program in Developmental Biology, The Hospital for Sick Children, and ${ }^{7}$ Department of Molecular and Medical Genetics, University \\ of Toronto, Toronto, Ontario, Canada M5G 1X8
}

Transcriptional programs guide the specification of neural cell types in the developing nervous system. However, it is unclear whether such programs also control specific aspects of neural circuit function at maturity. In the mammalian retina, Vs 1 and Irx 5 transcription factors are present in a subset of bipolar interneurons that convey signals from photoreceptors to ganglion cells. The biased expression of Vs $x 1$ and Ir $x 5$ in hyperpolarizing OFF compared with depolarizing ON bipolar cells suggests that these transcription factors may selectively regulate signal processing in OFF circuits. To test this hypothesis, we generated mice lacking both Vsx1 and Irx5. Bipolar cells in these mice were morphologically normal, but the expression of cell-specific markers in some OFF but not ON bipolar cells was reduced or absent. To assess visual function in $V s x 1^{-/-} \operatorname{Ir} x 5^{-/-}$retinas, we recorded light responses from ensembles of retinal ganglion cells (RGCs). We first identified functional RGC types in control mice and describe their response properties and adaptation to temporal contrast using a simple linear-nonlinear model. We found that space-time receptive fields of RGCs are unchanged in Vs $x 1^{-/-}$Ir $x 5^{-/-}$mice compared with control retinas. In contrast, response threshold, gain, and range were lowered in a cell-type-specific manner in OFF but not ON RGCs in $V s x 1^{-/-} \operatorname{Ir} x 5^{-/-}$retinas. Finally, we discovered that the ability to adapt to temporal contrast is greatly reduced in OFF RGCs in the double mutant, suggesting that $V s x 1$ and $I r x 5$ control specific aspects of visual function in circuits of the mammalian retina.

Key words: contrast adaptation; retina; transcription factor; bipolar cell; retinal ganglion cell; vision

\section{Introduction}

Reliable function of neuronal circuits depends on the physiological characteristics and precise connectivity of their cellular components. Genetic programs regulating the generation of neuronal cell types with distinct structure and function have been investigated extensively (Livesey and Cepko, 2001). In contrast, much less is known about how transcriptional programs regulate later stages of neuronal development and influence mature circuit function. In particular, it is unclear whether specific properties of neuronal circuits are under selective genetic control. Here, we investigated how the loss of two transcription factors normally

Received June 12, 2007; revised Dec. 31, 2007; accepted Jan. 4, 2008.

This work was supported by National Institutes of Health Grants (R.O.L.W.), Canadian Institutes of Health Research (R.L.C.), The Foundation Fighting Blindness (R.L.C.), the Research Grants Council of the Hong Kong Special Administrative region, China CityU 1474/05M (S.H.C.), and the Deutsche Forschungsgemeinschaft (D.K.). We thank members of the Wong laboratory for comments on this manuscript.

Correspondence should be addressed to either of the following: Rachel 0. L. Wong, Department of Biological Structure, University of Washington, Health Sciences Building, 1959 NE Pacific Street, Seattle, WA 98195, E-mail: wongr2@u.washington.edu; or Robert L.Chow, Department of Biology, University of Victoria, P.0. Box 3020, Station CSC, Victoria, British Columbia, Canada V8W 3N5, E-mail: bobchow@uvic.ca.

DOI:10.1523/JNEUROSCI.4784-07.2008

Copyright $\odot 2008$ Society for Neuroscience $\quad 0270-6474 / 08 / 282342-11 \$ 15.00 / 0$ present in a subclass of retinal bipolar cells impacts the lightresponse characteristics of their postsynaptic targets, the retinal ganglion cells (RGCs).

In rodents, at least 10 different types of retinal bipolar cells can be grouped into three subclasses (Ghosh et al., 2004): rod (one type), ON cone (five types), and OFF cone (four types) bipolar cells. Rod bipolar cells receive input selectively from rod photoreceptors, whereas $\mathrm{ON}$ and OFF cone bipolar cells primarily receive input from cones (Wassle, 2004). In addition to differences in connectivity, in response to light, ON cone and rod bipolar cells depolarize, whereas OFF cone bipolar cells hyperpolarize.

Retinal bipolar cell differentiation involves at least two stages of transcriptional control. First, expression of the homeobox transcription factor $C h x 10$ (Vsx2) and the basic helix-loop-helix $(b H L H)$ transcription factors Mash1 and Math3 in progenitor cells determine bipolar cell fate (Burmeister et al., 1996; Hatakeyama et al., 2001). A second tier of transcription factors is then thought to guide the specification of different bipolar cell types. For example, Bhlhb4 is required for the survival of rod but not cone bipolar cells (Bramblett et al., 2004), whereas Bhlhb5 is necessary for the generation of type 2 OFF cone bipolar cells (Feng et al., 2006). Recently, two homeobox transcription fac- 
tors, $V s x 1$ and the Iroquois gene Ir $x 5$, were found to be expressed in overlapping sets of mouse OFF cone bipolar cells (Chow et al., 2001; Cheng et al., 2005). Deletion of either gene did not affect the number or gross morphology of bipolar cells but led to distinct changes in the expression profile of the several OFF cone bipolar cell types (Chow et al., 2004; Cheng et al., 2005). In addition, ERGs of Vsx1-deficient mice showed a reduced b-wave, a measure of bipolar cell activity, and OFF but not ON RGCs fired on average fewer spikes in response to steps in light intensity compared with their wild-type counterparts (Chow et al., 2004; Ohtoshi et al., 2004). In contrast, preliminary studies revealed no specific b-wave defects in ERGs from Irx5-deficient mice (Cheng et al., 2005). These findings argued that Vsx1 and Ir $x 5$ regulate the differentiation of OFF cone bipolar cells and suggested their possible importance for retinal function. To determine whether specific properties of retinal circuit function are under the genetic control of these two transcription factors, we combined the knock-out of Vsx 1 and Irx 5 and measured light responses and adaptation to temporal contrast for ensembles of RGCs in double knock-out (dko) and control mice.

\section{Materials and Methods}

\section{Experimental procedures}

Mice. Mice that were heterozygous or null for $V s x 1$ and $I r x 5$ were bred from single gene knock-out mice of $V s x 1$ (Chow et al., 2004) and $I r x 5$ (Cheng et al., 2005) and genotyped using the following primer sets: Vsxl genotyping: common $5^{\prime}$ primer, TTC TAG GCT GTC TAG GTC TC; wild-type $3^{\prime}$ primer, TGA TGG CAA AGC TTC GAA GG; mutant 3' primer, TTG CCT TTA CTG ACC ATG CG; Irx5 genotyping: wild-type 5' primer, ACC GCT TCC TCG TGC TTT AC; mutant 5' primer, GCC ACC CAA AAG ACT GAA ACC; common 3' primer, TAA ACC TAT CTT CGC AAT CC. These mice were in a mixed 129/Sv and CD1 background. Throughout this study, double heterozygous animals were used as controls (see Results). However, to rule out that our identification of functional retinal ganglion cell types in mice was confounded by the genetic background of the mouse strain used, we initially compared light responses of retinal ganglion cells from a mixed 129/Sv and CD1 to those from a C57BL/J6 background. Results for these strains were indistinguishable (see Fig. 2).

Immunohistochemistry. Immunostaining was performed on retinal sections from 6-week-old mice fixed in 4\% paraformaldehyde/PBS overnight at $4^{\circ} \mathrm{C}$. For recoverin immunostaining, eyes were fixed for $30 \mathrm{~min}$ at room temperature. Tissue processing and immunostaining for frozen and paraffin sections were done as described previously (Chow et al., 2004; Cheng et al., 2005) with the following exceptions: sections were blocked in $10 \%$ horse serum, and primary antibodies were diluted in $1 \%$ horse serum. Primary antibodies used in this study were as follows: rabbit anti-recoverin (AB5585; Chemicon, Temecula, CA) (1:1000), guinea pig anti-Neto 1 (gift from R. McInnes, Hospital for Sick Children, Toronto, Ontario, Canada) (1:100), rabbit anti-NK3R (gift from Y. Ding, Chinese Acacemy of Sciences, Shanghai, China) (1:100), rabbit anti-CaB5 (gift from F. Haeseleer, University of Washington, Seattle, WA) (1:100), and rabbit anti-plasma membrane calcium transporting ATPase 1 (PMCA1) (PAI-914; Affinity BioReagents, Golden, CO) (1:100). Images were acquired using a Nikon (Tokyo, Japan) C1-plus laser scanning confocal Eclipse TE2000U inverted microscope.

Tissue preparation. Retinas were dissected from mice at ages ranging from postnatal day 80 (P80) to P100. Mice were dark adapted for at least $1 \mathrm{~h}$ before dissection, anesthetized with $5 \%$ halothane, decapitated, and enucleated under dim red illumination. The rest of the procedure was performed in a completely darkened room under infrared (IR) illumination using microscope-mounted IR converters (B. E. Meyers, Redmond, WA). Briefly, after enucleation, the cornea was punctured with a 30 gauge needle, and the eye was placed in cooled artificial CSF (ACSF) equilibrated with $95 \% \mathrm{O}_{2} 5 \% \mathrm{CO}_{2}$. ACSF contained the following (in mM): 125 $\mathrm{NaCl}, 2.5 \mathrm{KCl}, 1 \mathrm{MgCl}_{2}, 1.25 \mathrm{NaH}_{2} \mathrm{PO}_{4}, 2 \mathrm{CaCl}_{2}, 20$ glucose, and 26
$\mathrm{NaHCO}_{3}$. The retina was isolated, and $2-8 \mathrm{~mm}^{2}$ rectangular pieces were prepared at mid-eccentricity.

Multielectrode array recordings. The arrays used (HexaMEA; MultiChannelSystems, Reutlingen, Germany) comprised 60 electrodes arranged in a regular hexagon with electrode diameters $(10,20$, and $30 \mu \mathrm{m})$ and interelectrode distances $(30,60$, and $90 \mu \mathrm{m})$ increasing from center to periphery. Accordingly, the field of all electrodes covered an area of $\sim 0.2 \mathrm{~mm}^{2},>80 \%$ of the electrodes were contained within an area of $\sim 0.08 \mathrm{~mm}^{2}$, and $>50 \%$ of the electrodes were contained in an area of $\sim 0.04 \mathrm{~mm}^{2}$. The retina was transferred to a chamber containing the array, oriented ganglion cell side down and floated onto the electrode array. A transparent tissue culture membrane (Corning, Corning, NY) held down by a platinum ring was used to keep the retina in place. Retinas were allowed to settle on the array for at least $45 \mathrm{~min}$ before each recording as the amplitude of spikes typically increased during this period. The tissue was superfused continuously with ACSF (equilibrated with $95 \%$ $\mathrm{O}_{2} / 5 \% \mathrm{CO}_{2}$ ) at a rate of $\sim 1 \mathrm{ml} / \mathrm{min}$. The temperature of the bath was maintained at $31-33^{\circ} \mathrm{C}$. Signals were bandpass filtered between 100 and $3000 \mathrm{~Hz}$ and digitized at $10 \mathrm{kHz}$. Thresholds for spike detection were set manually for each channel using negative triggers. Spike cutouts from 1 ms before to $2 \mathrm{~ms}$ after threshold crossing and the time of threshold crossing were written to the hard disk and used for additional analysis. Typically, spikes from more than one cell were recorded at a given electrode. For each electrode, these spikes were sorted into trains representing the activity of single cells using principal component analysis of spike waveforms (Offline Sorter; Plexon, Denton, TX). The quality of the spike sorting was controlled by verifying refractory periods in the sorted trains. Only cells with $<0.2 \%$ interspike intervals of $<2$ ms were used for analysis. Often the activity of a single cell was recorded on multiple electrodes. In these cases, identified by cross-correlation of spike trains, only the recording with the cleanest spike sorting was retained.

Visual stimulation. Stimuli were presented on a monochrome white organic light emitting diode display (OLED; eMagin Corporation, Bellevue, WA; mean luminance at the retina $\sim 10^{5}$ photoisomerizations per middle-wavelength-sensitive cone per second, $\sim 10^{5} \mathrm{Rh}^{*} / \mathrm{M}$-cone/s; resolution, $800 \times 600$ pixels; $75 \mathrm{~Hz}$ vertical refresh) illuminating the back focal plane of a $10 \times$ objective (UPlanFLN; Olympus Optical, Tokyo, Japan) mounted on an inverted microscope (Axiovert S100; Zeiss, Jena, Germany) in place of the condenser and focused on the photoreceptors of the retina. Stimuli were programmed in Matlab (MathWorks, Natick, MA) using the Cogent graphics toolbox extensions (John Romaya, Laboratory of Neurobiology at the Wellcome Department of Imaging Neuroscience, University College London, London, UK). Checkerboard and full-field Gaussian white-noise stimuli were presented. For checkerboard stimulation, the intensity of squares of $66 \mu \mathrm{m}$ side length on the retina was chosen at random from a Gaussian distribution with constant mean (M) and SD every $40 \mathrm{~ms}$ (effective refresh rate, $25 \mathrm{~Hz}$ ). For full-field stimulation, similarly, intensity values were drawn every $40 \mathrm{~ms}$ from a Gaussian distribution. However, in this case, whereas the $\mathrm{M}$ of the distribution was constant, its SD was changed every $30 \mathrm{~s}$ to alternate the temporal contrast of the stimulus ( $c=\mathrm{SD} / \mathrm{M}$ ) between $40 \%$ (high contrast) and 20\% (low contrast). This interleaving of the stimulus safeguards against mistaking changes in the response during the course of an experiment for adaptation.

\section{Data analysis}

Model of light responses. We used a linear-nonlinear (LN) cascade model to describe the light responses of RGCs and compare response properties of different RGC types, of RGCs of the same type recorded from double knock-out and control mice, or the same cell stimulated at varying contrast levels. The model has been described in detail previously (Chichilnisky, 2001) and was shown to accurately predict much of the spiking of RGCs under similar stimulation and recording conditions (Chichilnisky, 2001; Kim and Rieke, 2001; Baccus and Meister, 2002; Zaghloul et al., 2003). Briefly, the first and linear stage of the LN model consists of a set of filters describing the spatial and temporal sensitivity profile of the cell, which are equivalent to its space-time receptive field. The stimulus is convolved with these filters, and the second stage of the model then describes how the filtered stimulus is transformed into the spiking re- 
sponse of RGCs. This transformation is generally nonlinear with rectification at low points of the filtered signal and saturation at its high points. The linear stage was recovered by correlating the stimulus to the response to compute the spike-triggered average (STA) stimulus. For checkerboard stimulation, we isolated the spatial and temporal aspects of the receptive field as follows. At the temporal maximum of the STA, a general two-dimensional Gaussian was fit to the spatial response profile. Similar to recordings from other species using comparable stimulation conditions (Devries and Baylor, 1997; Segev et al., 2006), receptive field surrounds were weak in our recordings, and fitting receptive field profiles with a difference of Gaussians offered no significant improvement and were therefore not included in the present analysis. The radius of the receptive field $(r)$ was defined as follows:

$$
r=\sqrt{r_{\text {min }} r_{\text {maj }}}
$$

where $r_{\min }$ and $r_{\text {maj }}$ signify the radius along the minor and major axis, respectively, of the 1-SD ellipse from Gaussian fits. The temporal structure of the receptive field center response was calculated by averaging the response of stimulus squares in the STA whose SD exceeded the SD of background pixels by a factor of more than three. Peak time and peakto-trough interval of temporal receptive fields were estimated from fits of the difference of two low-pass filters to these waveforms (Chichilnisky and Kalmar, 2002): $f(t)=a_{1}\left(t / \tau_{1}\right)^{n} e^{-n\left(t / \tau_{1}-1\right)}-a_{2}\left(t / \tau_{2}\right)^{n} e^{-n\left(t / \tau_{2}-1\right)}$, where $t$ is time before the spike, $f(t)$ is the normalized STA contrast at that time, and $a_{1}, a_{2}, n, \tau_{1}$, and $\tau_{2}$ are free parameters.

The filtered version of the stimulus (i.e., the generator signal) was calculated by convolving the stimulus with the STA. To avoid biasing LN model estimation, STA and generator signal were computed from separate parts of the recording. We assessed the dependence of the spike rate on the filtered stimulus by averaging the spike rate during time bins with similar generator signal values and used a standard normal cumulative distribution function (cdf) (Chichilnisky, 2001) to describe the average spike rate as a function of the generator signal according to $f(x)=$ $\alpha C(\beta(x-y))$. In this, $f(x)$ is the average spike rate, $x$ is the generator signal, $C(\ldots)$ is the cdf, $\alpha$ the maximum firing rate of the cell, $\beta$ the response gain, and $\gamma$ is the spike threshold. We used a Gauss-Newton algorithm with modifications for global convergence to find leastsquares parameter estimates.

Importantly, there is some ambiguity in the parameters of the LN cascade such that, if the STA and generator signal are both multiplied by a constant, the prediction of the model does not change. We chose to normalize the STA such that the variance of the generator signal equaled the variance of the stimulus (Baccus and Meister, 2002). Accordingly, the STA is used only to compare differences in the kinetics and spatial profile of the RGC light responses, whereas cell-specific differences or contrastdependent changes in their sensitivity are represented in the parameters describing the static nonlinearity. Several previous studies on contrast adaptation used a different kind of normalization in which horizontal scaling was used to best align the input/output relationship under high and low contrast stimulation (Chander and Chichilnisky, 2001; Kim and Rieke, 2001; Zaghloul et al., 2005). The same factor was then used to vertically scale the STA waveform. This has the advantage that both changes in kinetics and sensitivity can be visualized by comparing STAs under high and low contrast stimulation. However, in our recordings from mouse RGCs, similar to some recordings from rabbit and salamander (Baccus and Meister, 2002), in addition to changes in the sensitivity during contrast adaptation (i.e., horizontal scaling of input/ output relationships), we observed changes in the threshold of responses (i.e., horizontal shifts of input/output relationships). Thus, horizontal rescaling alone resulted in very poor overlap of input/output relationships under high and low contrast stimulation, and we chose instead to perform the normalization described above.

Statistics. Throughout this study, we used either Wilcoxon-MannWhitney rank sum tests or, in case of paired samples, signed rank tests to assess significance of differences between groups.

\section{Results}

We first determined the changes in the expression profile of retinal bipolar cells of mice lacking the transcription factors Vs $x 1$ and $\operatorname{Ir} \times 5$, both of which have been implicated previously in the late differentiation of OFF cone bipolar cells. We then recorded light responses from ensembles of RGCs in control mice and identified several functional RGC types and compared spacetime receptive fields, spiking nonlinearities, and adaptation to temporal contrast of the different RGC types across genotypes.

\section{Expression profile of bipolar cells in $V s x 1^{-/-} I r x 5^{-/-}$mice}

Previous studies identified both overlapping and nonoverlapping roles for $V s x 1$ and $\operatorname{Ir} x 5$ in the transcriptional regulation of type 2 and 3 OFF cone bipolar cells (Chow et al., 2004; Cheng et al., 2005) (supplemental Table S1, available at www.jneurosci.org as supplemental material). Similar to what was observed for $V s \times 1^{-1-}$ and $\operatorname{Ir} \times 5^{-/-}$mice, the retinas of $V s \times 1^{-/-} \operatorname{Ir} \times 5^{-/-}$(dko) mice appeared morphologically indistinguishable from wild type. To determine whether bipolar cells were generated in normal numbers, Chx10-positive cells were counted in retinal cross sections labeled with the nuclear dye TO-PRO-3. This confirmed that bipolar cells make up $58 \pm 2 \%(n=5$ retinas $)$ and $59 \pm 3 \%$ ( $n=6$ retinas) of cells in the inner nuclear layer of wild-type and dko mice, respectively. To validate that, among them, type $2 \mathrm{OFF}$ cone bipolar cells form in normal numbers, we counted the fraction of $\beta$-galactosidase ( $\beta$-gal)-positive cells among Chx10positive cells in dko mice (in which $\beta$-gal expression is driven by the Vsx 1 promoter $)(18.7 \pm 1.3 \% ; n=4$ retinas $)$ and compared it with the fraction of Vsx1-positive cells among Chx10-positive cells in wild-type littermates ( $17.1 \pm 1.1 \% ; n=2$ retinas). Thus, bipolar cell types normally expressing Vsxl appear to be present in wild-type numbers in the dko mice.

Next, we studied the expression profiles of bipolar cells in these mice. Retinas were stained for cell-specific markers of different bipolar cell types (Fig. 1) (supplemental Table S1, available at www.jneurosci.org as supplemental material). Although in both the $V s x 1^{-1-}$ and Ir $x 5^{-/-}$mice the portion of recoverinpositive cells among type 2 OFF cone bipolar cells is reduced (Chow et al., 2004; Cheng et al., 2005), we found recoverin labeling to be altogether absent from type 2 OFF cone bipolar cells of dko mice (Fig. $1 B$ ). In addition, the fraction of Netol-positive cells among type 2 cells was reduced in dko mice (Fig. $1 D$ ) in a manner comparable with the $V s x 1^{-/-}$single mutant (Chow et al., 2004). NK3R, another marker of type 2 cells, labeled a subset of bipolar cells indistinguishable in morphology and number to wild-type mice but showed lower expression levels in those cells. Thus, type 2 OFF cone bipolar cells appear to be present in normal numbers in dko mice, but their expression profiles differ from wild type.

Ir $x 5^{-/-}$mice showed a loss of calcium-binding protein 5 (CaBP5) and PMCA1 expression in type 3 OFF cone bipolar cell axon terminals (Cheng et al., 2005). Although Vsxl itself could not be detected in type 3 OFF cone bipolar cells, CaBP5 immunolabeling, but not PMCA1, of the type 3 axon terminals was reduced in the $V s \times 1^{-/-}$mice (Chow et al., 2004). Figure 1 shows that, as in $\operatorname{Ir} x 5^{-/-}$mice, type 3 OFF cone bipolar cell axon terminals of dko mice lack both CaBP5 and PMCA1 labeling (Fig. $1 H, J, L)$. Similar to the reduced rather than absent expression of NK3R in type 2 cells, the restriction of the defect of CaBP5 and PMCA1 expression to axon terminals of type 3 cells allowed us to infer that these cells are present in regular numbers in dko mice but differ from cells in wild-type mice in the expression of several proteins.

In summary, dko mice appear to have accumulated the defects in expression of cell-specific markers in OFF but not ON cone bipolar cells from $V s x 1^{-/-}$and $\operatorname{Ir} x 5^{-/-}$mice. However, the num- 


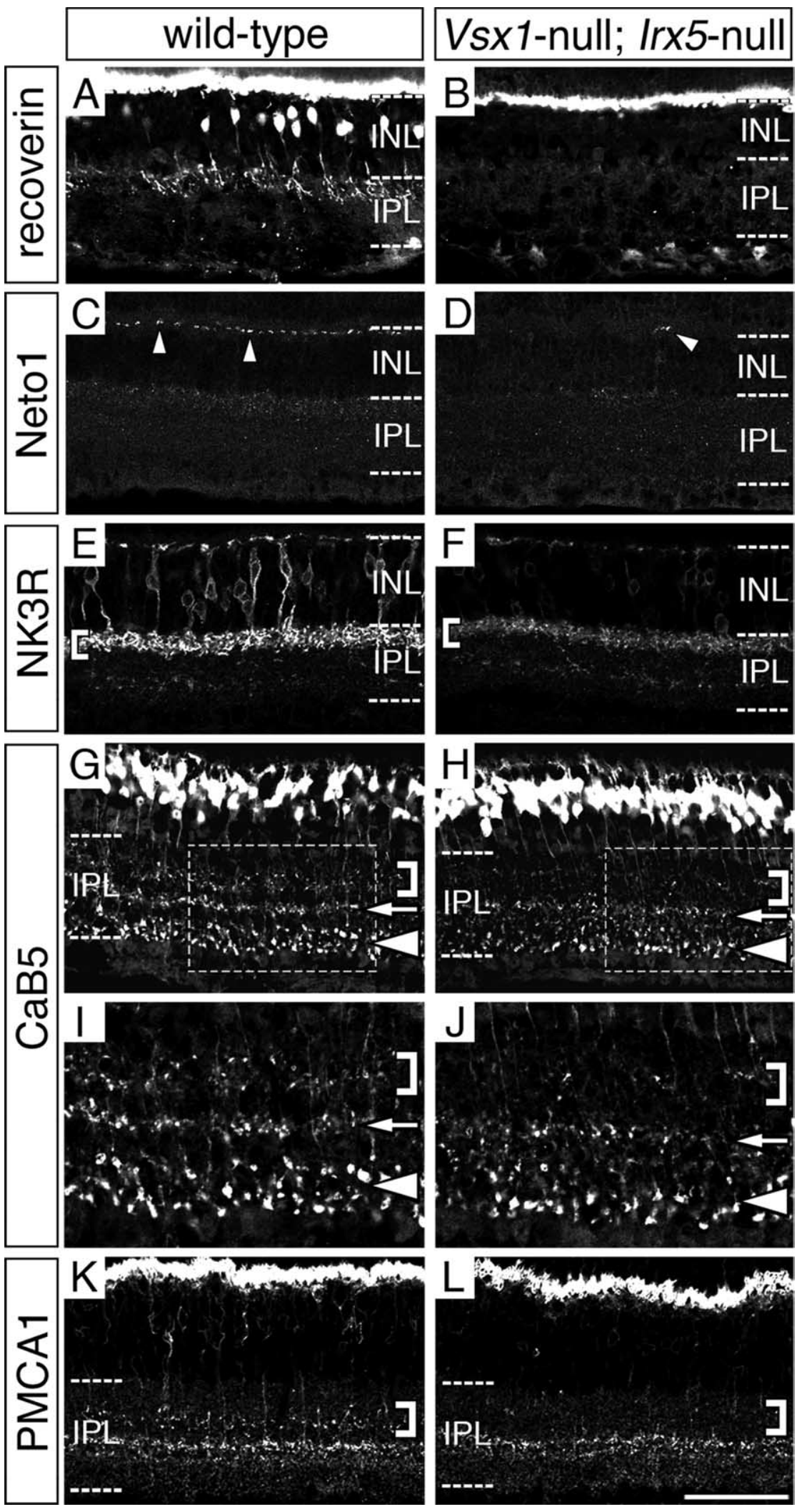

Figure 1. Expression profiles of bipolar cells in retinas of dko and wild-type mice. Immunolabeling of retinal sections from 6-week-old wild-type $(\boldsymbol{A}, \boldsymbol{C}, \boldsymbol{E}, \boldsymbol{G}, \boldsymbol{I}, \boldsymbol{K})$ and dko $(\boldsymbol{B}, \boldsymbol{D}, \boldsymbol{F}, \boldsymbol{H}, \boldsymbol{J}, \boldsymbol{L})$ mice with markers for OFF $(\boldsymbol{A}-\boldsymbol{F})$ or $0 \mathrm{~N}$ and $\mathrm{OFF}(\boldsymbol{G}-\boldsymbol{L})$ bipolar cells. Boundaries of inner nuclear layer (INL) and the inner plexiform layer (IPL) are indicated. Arrowheads in $C$ and $\boldsymbol{D}$ point to Neto 1 labeling in the dendrites of type 2 OFF cone bipolar cells. G-J, Brackets highlight laminar depth of CaBP5 labeling in type 3 OFF cone bipolar cell axonal terminals, arrows show CaBP5 staining in type $50 \mathrm{~N}$ cone bipolar cell terminals, and arrowheads point to CaBP5-positive rod bipolar cell terminals. $\boldsymbol{K}, \boldsymbol{L}$, Brackets indicate the part of the inner plexiform layer normally occupied by type $30 \mathrm{FF}$ cone bipolar cell axon terminals. Scale bar, $50 \mu \mathrm{m}$ for all panels, except $I$ and $J(23 \mu \mathrm{m})$. ber and gross morphology of cells generated as well as the lamination of their axon terminals in the inner plexiform layer appears indistinguishable from that of wild-type retinas.

\section{Identification of functional RGC types} in mice

To evaluate retinal function in mice lacking $V s \times 1$ and $\operatorname{Ir} \times 5$, we simultaneously recorded spike trains from ensembles of RGCs using multielectrode arrays while presenting a randomly flickering white-noise checkerboard stimulus. We then described the transformation from stimulus to spiking of RGCs using a simple LN model (Chichilnisky, 2001). To allow for meaningful comparison of results from dko and control mice, it was first necessary to identify functional types of mouse RGCs in our recordings. The left column of Figure 2 shows the temporal structure of STAs from representative recordings from wild-type mice in a C57BL/J6 background (Fig. $2 A$ ) and $V s x 1^{+/-} \operatorname{Ir} x 5^{+/-}$(Fig. $2 B$ ) and dko mice (Fig. 2C) in a mixed $129 / \mathrm{Sv}$ and CD1 background. STAs were calculated from reverse correlation of the spike train to checkerboard stimulation. Cells were clustered into distinct types using agglomerative $k$-means algorithms on the temporal structure of the STAs (Segev et al., 2006). Approximately 80\% of all cells recorded ( 137 of 169 cells; $n=10$ retinas) belonged to one of four functional types identified in this way. Among the remaining unclassified cells were presumably direction-selective cells that showed inseparable space-time receptive fields in which the peak of the response moved across the retinal surface during the time preceding a spike and sluggish ON and OFF cells. Some sluggish OFF cells showed small receptive field centers and weak responses to full-field stimulation, which are features characteristic of local edge detector cells originally characterized in rabbit (Levick, 1967; Devries and Baylor, 1997) and recently identified in mice (Zeck et al., 2005). Responses of these unclassified cells were not analyzed further because of the small sample size that prevented comparison between genotypes.

The four cell types we analyzed in detail are referred to as fast $\mathrm{ON}$, medium $\mathrm{ON}$, biphasic OFF, and monophasic OFF RGCs based on the waveforms describing their temporal receptive fields. We tested the validity of this classification by including other aspects of the light response into the clustering procedure. This was achieved by making vectors representing the respective parameters unit length, before concatenating them and subjecting them to $k$-means clustering. Inclusion of the autocorrelation function and/or responses to diffuse light steps did not change 
our classification (Segev et al., 2006). Instead, it revealed that responses of fast $\mathrm{ON}$ cells were more sustained than for medium ON cells, and responses of biphasic OFF cells were more sustained compared with those of monophasic OFF cells (data not shown). It is worth noting that we do not mean to suggest that this classification of RGCs is comprehensive. As stated above, several unclassified cells, some with properties similar to cell types identified previously in other studies, were recorded, and likely several types were missed because of sampling of the multielectrode array. Our interest in the present study was only to reliably identify and characterize a set of functional cell types in our recordings to allow for a comparison of RGC light responses between control and dko mice.

To illustrate how receptive fields of ganglion cells of a given type were distributed on the retinal surface, we plotted the 1-SD ellipses obtained by fitting Gaussian surfaces to their spatial response profiles (Fig. $2 B, D, F)$. In these plots, receptive fields of the more frequently recorded fast $\mathrm{ON}$ and biphasic OFF cells appear to tile the retina, and their centers spaced uniformly and their 1-SD contours approached neighboring cells of the same type (for quantification, see supplemental Fig. S1, available at www.jneurosci.org as supplemental material). This was true for recordings from all genotypes. It is unlikely that the regular distribution of receptive fields is an artifact of the arrangement of recording electrodes on the array, because the electrodes are spaced irregularly with increasing interelectrode distances from the center to the border of the array. In addition, spikes from a given cell, in particular when recorded on electrodes in the center of the array, were usually detected by multiple electrodes, making it improbable that a large number of additional cells of the same type were missed. We therefore take the regular arrangement of spatial receptive fields as additional support of our classification of RGCs based on their temporal receptive fields.

As illustrated by the traces from representative recordings, no striking differences were observed between space-time receptive fields of RGCs in mice in a C57BL/J6 or mixed 129/Sv and CD1 background, demonstrating that the same types of RGCs were identified independent of the strain (Fig. 2). However, because the expression profiles of bipolar cells from $V s \times 1^{+/-}$Ir $x 5^{+/-}$mice were indistinguishable from wild-type mice in the same background (data not shown), and ERG recordings were normal for mice heterozygous for either Vsx1 or Irx5 (Chow et al., 2004; Cheng et al., 2005), we restricted our analysis for the remainder of this study to comparing retinal network function from dko and $V s \times 1^{+/-}$Ir $x 5^{+/-}$(control) mice that are in the same strain background.
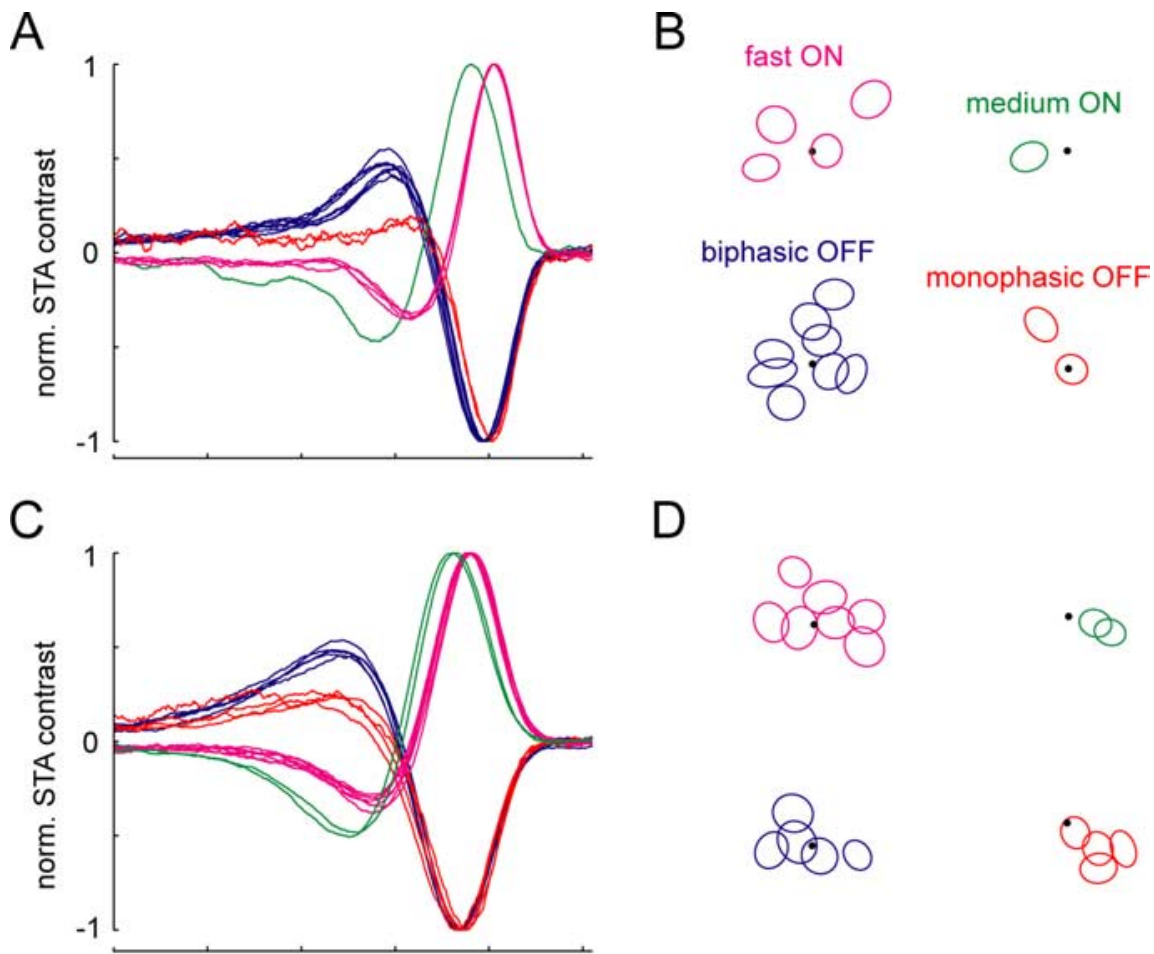

D

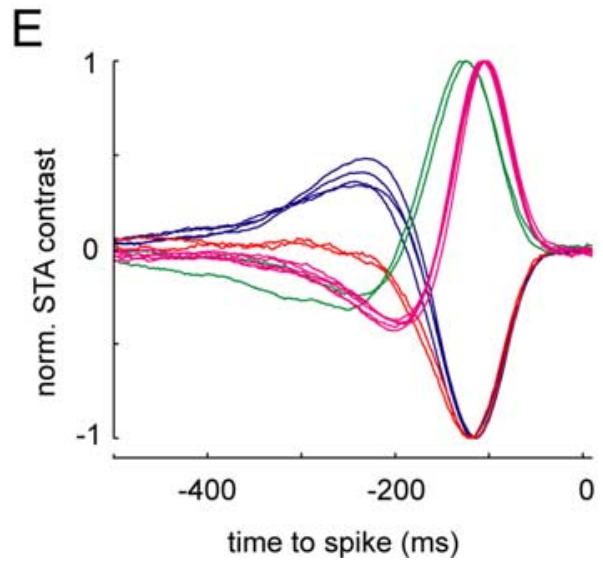

F
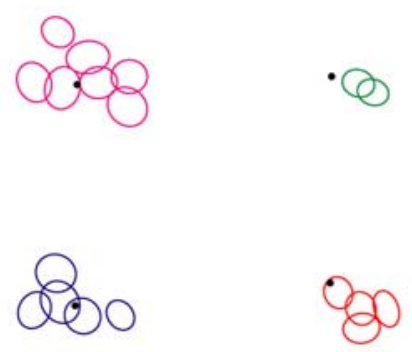

Figure 2. Temporal and spatial $\mathrm{RGC}$ receptive field maps of representative recordings from wild-type $(\boldsymbol{A}, \boldsymbol{B})$ ${\mathrm{V} s \times 1^{+/-}} \mathrm{rx} \times 5^{+/-}(\boldsymbol{C}, \boldsymbol{D})$ and dko $(\boldsymbol{E}, \boldsymbol{F})$ mice. RGC light responses were recorded in mice aged P80-P100. $\boldsymbol{A}, \boldsymbol{C}, \boldsymbol{E}$, Temporal or dko $(\boldsymbol{E}, 13 \mathrm{RGCS})$ retina are shown. Spike-triggered average waveforms in these plots were normalized to their amplitude and color coded to indicate fast ON (magenta), medium ON (green), biphasic OFF (blue), and monophasic OFF (red) RGCS. B, D, $\boldsymbol{F}_{\text {, }}$ contours of two-dimensional Gaussian fits to receptive field profiles. Receptive field contours are color coded as indicated, and plots of the different cell types are offset for visual clarity. Dots represent a common point on the multielectrode array.

\section{RGC space-time receptive fields of different types and genotypes}

Similar to previous studies using comparable recording techniques in rabbit and monkey, receptive field parameters showed considerable inter-experiment variation for cells of a given type, whereas light responses of cells of the same type recorded simultaneously were closely matched (Devries and Baylor, 1997; Chichilnisky and Kalmar, 2002). In addition, the relative differences in space-time receptive fields of the various RGC types were consistent across experiments. Figure 3 shows that the integration time of fast $\mathrm{ON}$ cells was consistently shorter than that of medium ON cells (Fig. 3A, left). Accordingly, the time-to-peak determined from the temporal receptive field structure was $13 \pm$ 


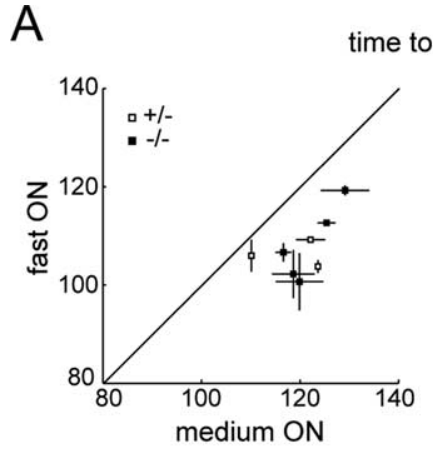

time to peak (ms)

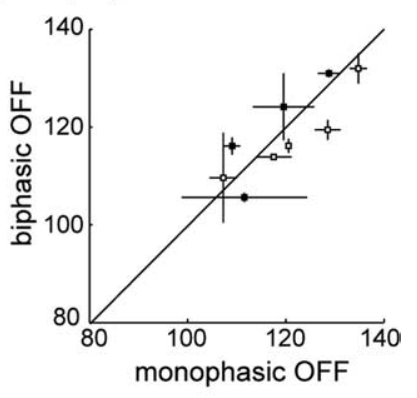

B
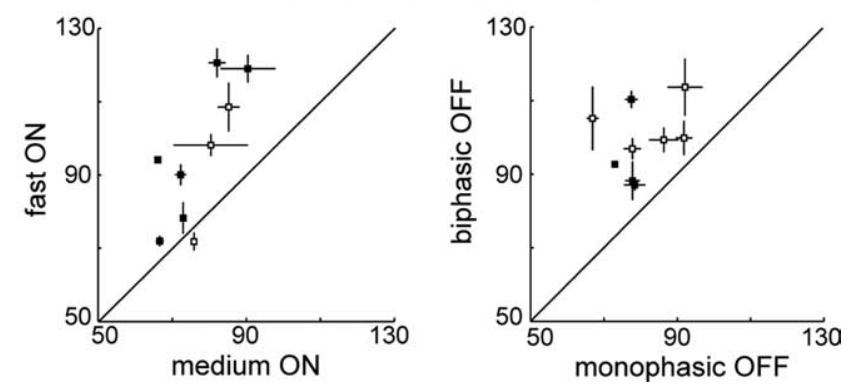

Figure 3. Comparison of space-time receptive field parameters of the different RGC types from control and dko mice. $\boldsymbol{A}$, Plot of the time by which the peak modulation of the temporal receptive field precedes spiking of $0 \mathrm{~N}$ (left) and $0 \mathrm{FF}$ (right) cells. $x$-Axis corresponds to peak time for medium ON RGCs (left) and monophasic OFF RGCs (right). Values on the $y$-axis indicate peak time for fast ON RGCs (left) and biphasic OFF RGCs (right). Each square (error bars) represents the mean \pm SEM for all cells of the respective types recorded in one experiment. Only experiments in which at least three cells of the respective types were recorded were plotted in these graphs. Data from experiments on control mice $\left(+/-\right.$, i.e., $\left.V s \times 1^{+/-} I r \times 5^{+/-}\right)$and dko mice $(-/-$, i.e., $V s \times 1^{-/-} I r \times 5^{-/-}$) are marked by open and filled symbols, respectively. $\boldsymbol{B}$, Plot of receptive field radii for the different cell types and genotypes. Axis orientation and symbols as in $\boldsymbol{A}$. Likewise, as in $\boldsymbol{A}$, each square (error bars) represents the mean \pm SEM from one experiment.

$2 \%$ (mean \pm SEM) shorter for fast ON cells compared with medium ON cells for both genotypes $(p<0.004)$. By comparison, no such difference was apparent between biphasic and monophasic OFF cells (Fig. $3 A$, right) ( $p>0.8$ ). The relationship of temporal receptive fields of cells from different types was identical for recordings from dko and control mice, and the mean time-topeak recorded in different experiments varied over a common range.

To evaluate the spatial extent of receptive fields, we compared the receptive field radii (see Materials and Methods). Receptive fields of fast ON cells were $23 \pm 6 \%$ larger than those of medium ON RGCs $(p<0.04)$, and receptive fields of biphasic OFF cells were $25 \pm 5 \%$ larger than those of monophasic OFF cells $(p<$ 0.004) (Fig. 3B). Again, results were indistinguishable for recordings from both genotypes. To ensure that we did not artificially generate differences in the receptive field size by fitting Gaussian profiles, we used the number of pixels whose SD during the STA exceeded the SD of background pixels by a factor of more than 3 as a nonparametric indicator of receptive field extent (data not shown). This analysis confirmed the asymmetry in receptive field size between fast $\mathrm{ON}$ and medium $\mathrm{ON}$ as well as between biphasic OFF and monophasic OFF cells. Finally, several aspects of their space-time receptive fields suggests that fast $\mathrm{ON}$ and biphasic OFF, as well as medium ON and monophasic OFF may each be $\mathrm{ON}$ and $\mathrm{OFF}$ variants of the same functional type of RGC. In both cases, ON and OFF cells were recorded at a similar frequency, arguing that the electrotonic properties leading to their sampling are comparable. In addition, the temporal structure of their receptive fields were approximate opposite sign versions of the same waveform, their autocorrelation functions were similar, and their receptive field radii were indistinguishable.

\section{RGC spiking nonlinearities and contrast sensitivity for different cell types and genotypes}

To compare gain, threshold, and range of RGC light responses using an LN model, we normalized STA amplitudes so that the variance of the generator signal (i.e., stimulus weighted by the STA) equaled the variance of the stimulus (Baccus and Meister, 2002) (see Materials and Methods). Figure 4 shows the dependence of the spiking response on the generator signal for the different RGC types from dko and control mice. Comparison of fast ON (Fig. 4A) and biphasic OFF (Fig. 4C) cells revealed asymmetries between $\mathrm{ON}$ and $\mathrm{OFF}$ pathways. Compared with fast $\mathrm{ON}$ cells, the spiking response of biphasic OFF cells showed 159\% higher threshold and 79\% higher gain $(p<0.001)$, which are evident from the right shift and steeper rise, respectively, of their contrast response curves (Fig. $4 A, C$, left). In addition, responses from biphasic OFF cells had greater range (i.e., maximum spike rate, $53 \pm 3 \mathrm{~Hz})$ compared with responses of fast $\mathrm{ON}(32 \pm 1 \mathrm{~Hz})$ cells. Interestingly, the response range was greater for medium $\mathrm{ON}(32 \pm 3 \mathrm{~Hz})$ compared with monophasic OFF $(19 \pm 2 \mathrm{~Hz})$ cells $(p<0.01)$, whereas their gain and threshold was not significantly different $(p>0.6)$, arguing that $\mathrm{ON}-\mathrm{OFF}$ asymmetries are cell type specific rather than general between both pathways. In particular, medium ON cells showed similarly strong rectification at low generator signals as did both types of OFF cells analyzed. For both fast and medium ON cells, results from dko and control mice were indistinguishable (Fig. $4 A, B$ ). In contrast, biphasic OFF cells from dko mice showed $8 \%$ lower threshold and $23 \%$ lower gain than in control mice $(p<0.03)$ (Fig. $4 C)$. In addition, the output range of these cells was reduced by $38 \%(p<$ 0.001 ) and was paralleled by reduced responses of these cells to full-field stimulation (supplemental Fig. S2, available at www. jneurosci.org as supplemental material). Interestingly, these effects were cell type specific because monophasic OFF cells from dko mice showed reduced threshold $(18 \% ; p<0.001)$ and gain $(44 \% ; p<0.001)$ but normal range $(p>0.9)$ (Fig. $4 D)$.

\section{Temporal contrast adaptation in responses of RGCs from different types and genotypes}

To assess contrast adaptation in RGCs, we presented a flickering full-field stimulus whose intensity was randomly chosen every 40 ms from a Gaussian distribution. The width of the distribution was alternated every $30 \mathrm{~s}$, such that the temporal contrast of the stimulus varied between $40 \%$ (high contrast) and 20\% (low contrast). Typically, the average spike rate of RGCs declined and recovered gradually over several seconds, following steps to high and low contrast, respectively (data not shown), indicating that contrast adaptation in mice, similar to other species, has a slow component (Smirnakis et al., 1997; Chander and Chichilnisky, 2001; Kim and Rieke, 2001; Baccus and Meister, 2002; Manookin and Demb, 2006). For this study, we discarded responses from the first $15 \mathrm{~s}$ after each contrast step to allow adaptation to approach steady state. By calculating the parameters of the LN model for responses from the different contrast segments, one can separate adaptation from instantaneous spiking nonlinearities that would otherwise confound an analysis of contrastdependent changes to the light response of a cell.

Figure 5 shows examples of contrast adaptation for fast $\mathrm{ON}$ (Fig. $5 B, D$ ) and biphasic OFF (Fig. 5C,E) cells from control (Fig. 
$5 B, C)$ and dko (Fig. 5D,E) mice. For both $\mathrm{ON}$ and OFF cells of either genotype, temporal receptive fields broadened and sensitivity increased in response to low contrast stimulation. Because we normalized the amplitude of the STA, all changes in response sensitivity are depicted by the static nonlinearity. To evaluate the gain increase in response to stimuli of lower contrast in these examples, we first calculated the average gain adaptation (i.e., the ratio of gain under low and high contrast stimulation) for all cells of a given type recorded in control mice and then derived the expected input/output relationship (Fig. $5 B-E$, solid lines in right panels) by multiplying the gain under high contrast stimulation of each example cell with this ratio. This revealed that the gain increase in the responses of biphasic OFF cells during adaptation to stimuli of lower contrast was reduced in dko compared with control mice (Fig. 5C,E). Conversely, no differences in the contrast adaptation of fast $\mathrm{ON}$ cells from control and dko mice were apparent in these examples.

\section{Changes in response kinetics during contrast adaptation}

To quantify the changes to response kinetics that accompany contrast adaptation and compare them for recordings from dko and control mice, we measured the time-to-peak of STAs at high and low contrast. This revealed that response kinetics were sped up during high contrast stimulation by $4.3 \pm 0.4 \%(p<0.001)$ and $5 \pm$ $0.2 \%(p<0.001)$ for $\mathrm{ON}$ cells from control and dko mice, respectively (Fig. $6 A$, left). Values obtained from the different cell types and genotypes were not significantly different $(p>0.07)$. Similarly, OFF cell kinetics were accelerated by $5.6 \pm 0.6 \%$ $(p<0.001)$ for control and by $4.4 \pm 0.4 \%$ $(p<0.001)$ for dko mice with no significant differences between these groups $(p>0.1)$ (Fig. $6 A$, right).

STA waveforms of fast and medium $\mathrm{ON}$ as well as biphasic OFF cells were clearly biphasic (Fig. 2). Thus, to examine whether the temporal receptive fields were compressed, we measured the interval from the trough to the peak of these waveforms during high and low contrast stimulation. For monophasic OFF cells, we measured the rise time from 5\% of the STA amplitude to its peak. This showed that the integration time of these neurons was significantly shortened during high contrast stimulation (ON cells control, $5 \pm 0.1 \%$; ON cells dko, $6 \pm 1 \%$; OFF cells control, $13 \pm 3 \%$; OFF cells dko, $15 \pm 2 \%)$. Similar to the results for the peak time (Fig. 6A), compression was significant for all RGC types $(p<$ $0.001)$, and differences between control and dko mice were insignificant $(p>0.1)$.

Changes in static nonlinearity during contrast adaptation Previous studies in other species have found that RGCs increase their sensitivity in response to low contrast stimulation (Sakai et
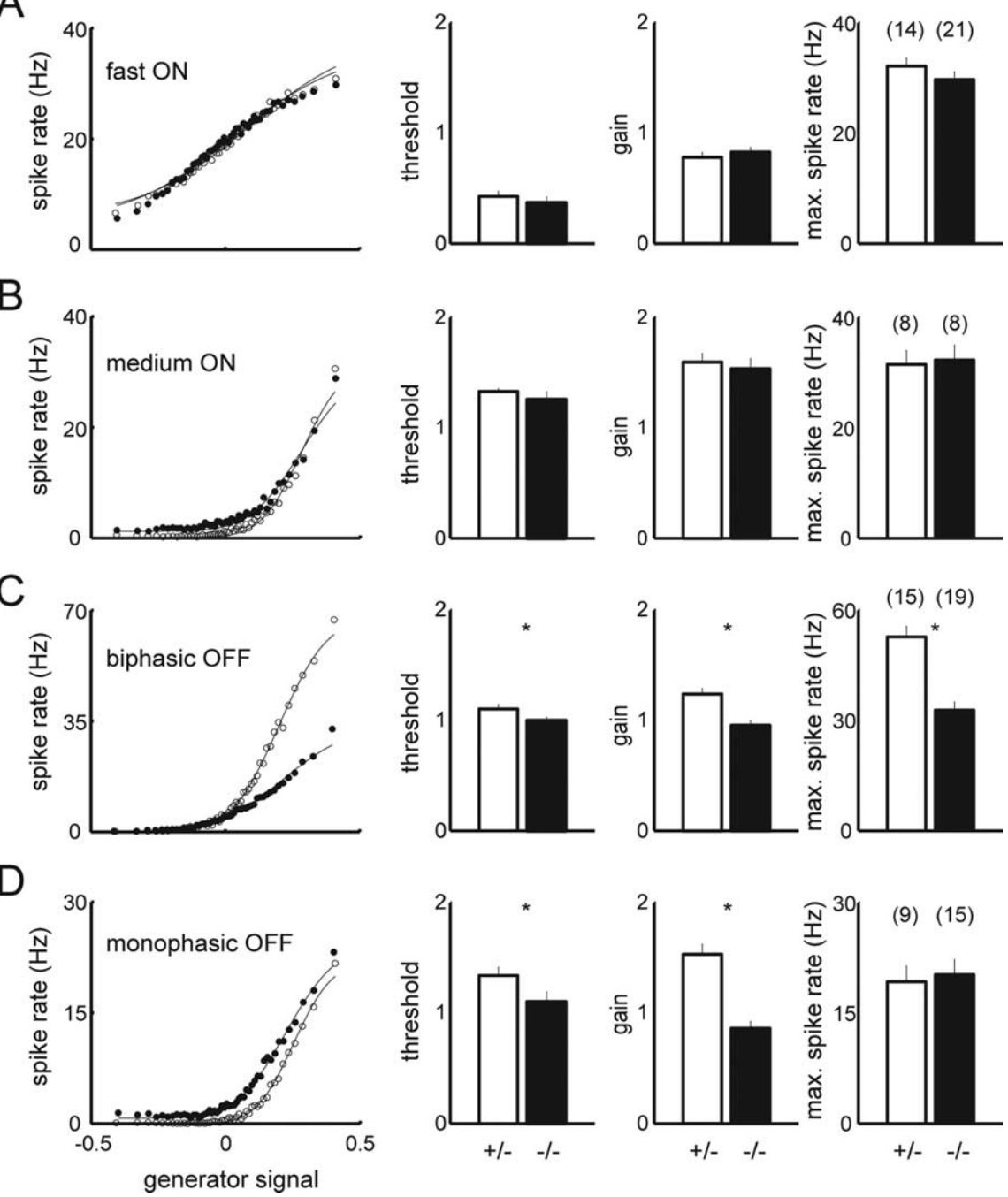

Figure 4. Comparison of static nonlinearities for RGCs of the different cell and genotypes. Left panels show firing rate as a function of the generator signal for representative fast ON $(\boldsymbol{A})$, medium $0 \mathrm{~N}(\boldsymbol{B})$, biphasic OFF $(\boldsymbol{C})$, and monophasic OFF $(\boldsymbol{D})$ RGCS of the response threshold, gain, and range for all cells of the respective types recorded from control (open bars) or dko (filled bars) mice. Numbers in brackets signify number of cells analyzed. * $p<0.05$ using Wilcoxon-Mann-Whitney rank sum tests.

al., 1995; Smirnakis et al., 1997; Chander and Chichilnisky, 2001; Kim and Rieke, 2001; Zaghloul et al., 2005). When we calculated the static nonlinearities of mouse RGCs under high and low contrast stimulation, we found that the response threshold is lowered under low contrast stimulation by $74 \pm 3 \%$. This effect was indistinguishable for the different cell types and for recordings from control and dko mice ( $p>0.1$, for 100 cells from 8 retinas). In contrast, increases in the response gain during low contrast stimulation differed significantly for the different RGC types and were reduced drastically in OFF but not ON cells of dko compared with control mice (Fig. 7). We used the ratio of response gain under low and high contrast stimulation (Fig. $7 B$, adaptation index) to quantify these differences. We found that medium ON cells of either genotype adapted more strongly than fast ON cells (Fig. $7 B$, left $)(p<0.02)$. Both this trend and the level of adaptation were indistinguishable for results from control and dko mice $(p>0.3)$. Biphasic and monophasic OFF RGCs, conversely, showed similar gain adaptation. Strikingly, gain adaptation was greatly reduced for both OFF RGC types in dko compared with control mice $(p<0.002)$ (Fig. $7 B$, right). 

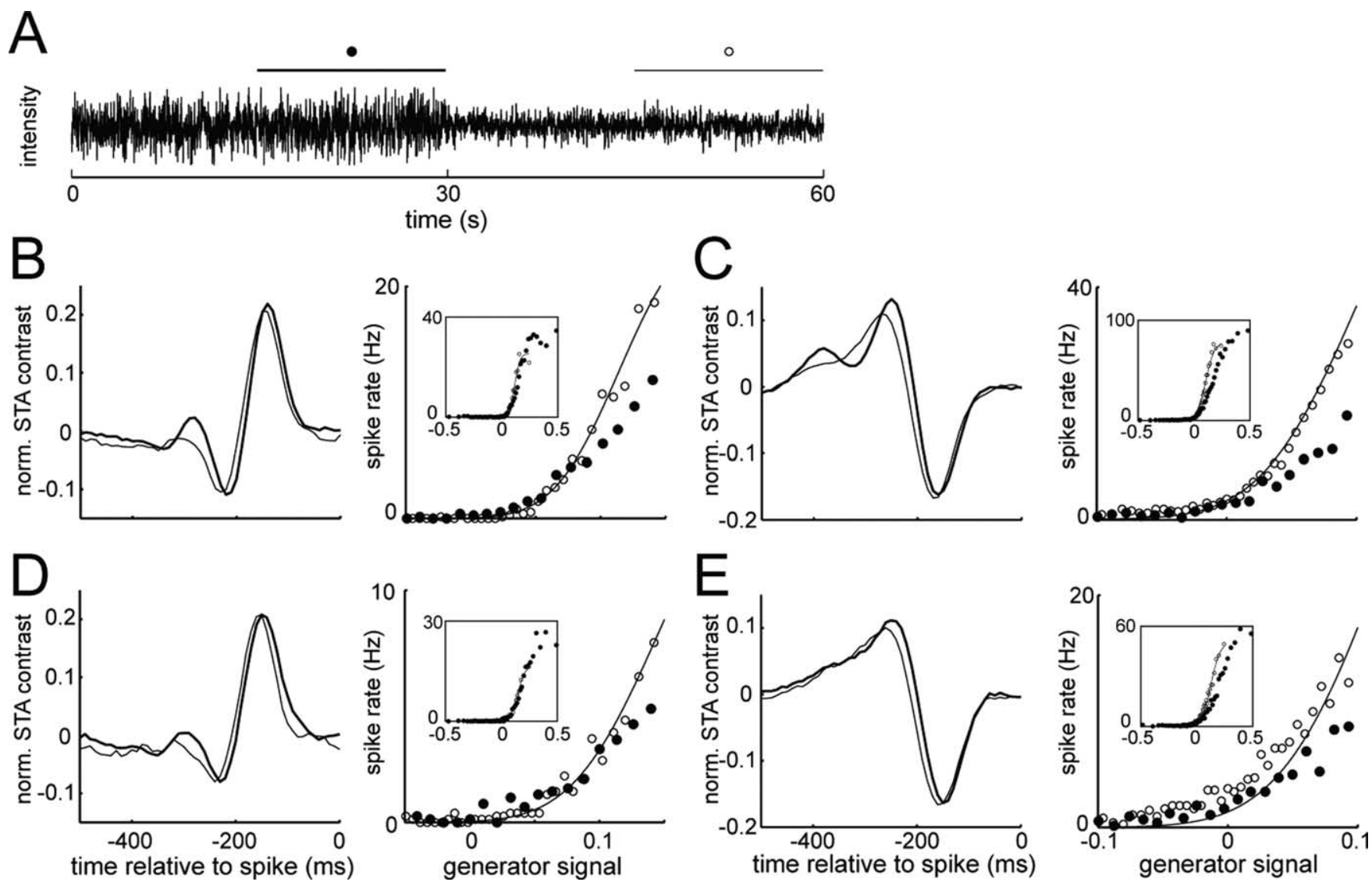

Figure 5. Contrast adaptation in the $L N$ model for representative $O N$ and $O F F ~ R G C s$ of control and dko mice. Temporal contrast of a randomly flickering full-field stimulus was altered every $30 \mathrm{~s}$ $(\boldsymbol{A})$, and responses were reconstructed from the second half of each contrast segment to allow adaptation to approach steady state. $\boldsymbol{B}-\boldsymbol{E}$, Linear filter and static nonlinearity of representative fast ON $(\boldsymbol{B}, \boldsymbol{D})$ and biphasic OFF $(\boldsymbol{C}, \boldsymbol{E})$ cells from control $(\boldsymbol{B}, \boldsymbol{C})$ or dko $(\boldsymbol{D}, \boldsymbol{E})$ mice during high (bold line, filled circles) and low contrast (thin line, open circles) segments of the stimulus. For plots of the static nonlinearity, full input/output relationships are shown as insets with enlargements of the foot of the respective curves as the main panel. In these plots, the expected low contrast input/output relationship of each cell is shown as a line. It is derived by using the average gain increase of all control cells of the respective type under low contrast stimulation together with the actual threshold and range of the cell. This shows that, for fast $\mathrm{ON}$ cells in control and dko and for biphasic OFF cells in control mice, gain adaptation fits the expectation, whereas the shallower rise of the data points (open circles in $\boldsymbol{E}$ ) compared with the expectation (solid line) indicates reduced gain adaptation of biphasic $0 \mathrm{FF}$ cells in dko mice.

\section{Discussion}

Our study contributes the following main findings. First, we identified several functional RGC types in mice, analyzed their space-time receptive fields, response threshold, gain, and range, and determined how these parameters change during adaptation to temporal contrast. We then used these parameters to ask whether specific aspects of retinal network function are under selective genetic control of the transcription factors Vsxl and Irx 5, which regulate expression profiles of OFF cone bipolar cells (Chow et al., 2004; Ohtoshi et al., 2004; Cheng et al., 2005). We found that space-time receptive fields are indistinguishable between genotypes and that differences in response threshold, gain, or range in retinas from dko compared with control mice are cell type specific and restricted to the OFF but not the ON pathway. Finally, we discovered that the ability to adapt to temporal contrast is greatly reduced in both types of OFF RGCs analyzed in retinas lacking $V s \times 1$ and $I r x 5$, arguing that these transcription factors control a crucial aspect of visual function in the retina.

Light response properties of RGCs in control and dko mice Different aspects of an image are processed in the retina in distinct, primarily parallel channels, whose information is passed on to the brain by different types of RGCs (Wassle, 2004). Thus, to analyze visual function of the retina and compare it between control and dko mice, we first needed to identify functional RGC types in our recordings. For each recording, cells were segregated by quantitative clustering based on their temporal receptive fields. In this way, $\sim 80 \%$ of the cells we recorded fell into four groups: fast and medium ON cells and biphasic and monophasic OFF cells. Several observations support the identification of these RGC types. First, simultaneously recorded cells of a given type were regularly spaced across the retinal surface with little overlap between adjacent receptive field profiles (Fig. 2) (supplemental Fig. S1, available at www.jneurosci.org as supplemental material). Second, grouping of cells according to these types identified robust differences in spatial receptive fields, spiking nonlinearity, and contrast adaptation, which were not part of the clustering procedure. We tentatively propose that the four RGC types analyzed represent $\mathrm{ON}$ and OFF pairings of two functional types. This pairing of fast $\mathrm{ON}$ and biphasic OFF, and medium $\mathrm{ON}$ and monophasic OFF is based on the similar frequency of these cells in our recordings, suggesting similar electrotonic properties and their comparable and opposite sign space-time receptive fields. Similar to what has been observed for monkey parasol and guinea pig Y cells, fast ON cells showed more linear input/output relationships than biphasic OFF cells, which showed strong rectification at low effective contrasts (i.e., low generator signal values) (Chichilnisky and Kalmar, 2002; Zaghloul et al., 2003). This ONOFF asymmetry, which has been suggested to underlie the higher perceptual sensitivity to low contrast decrements (signaled by 


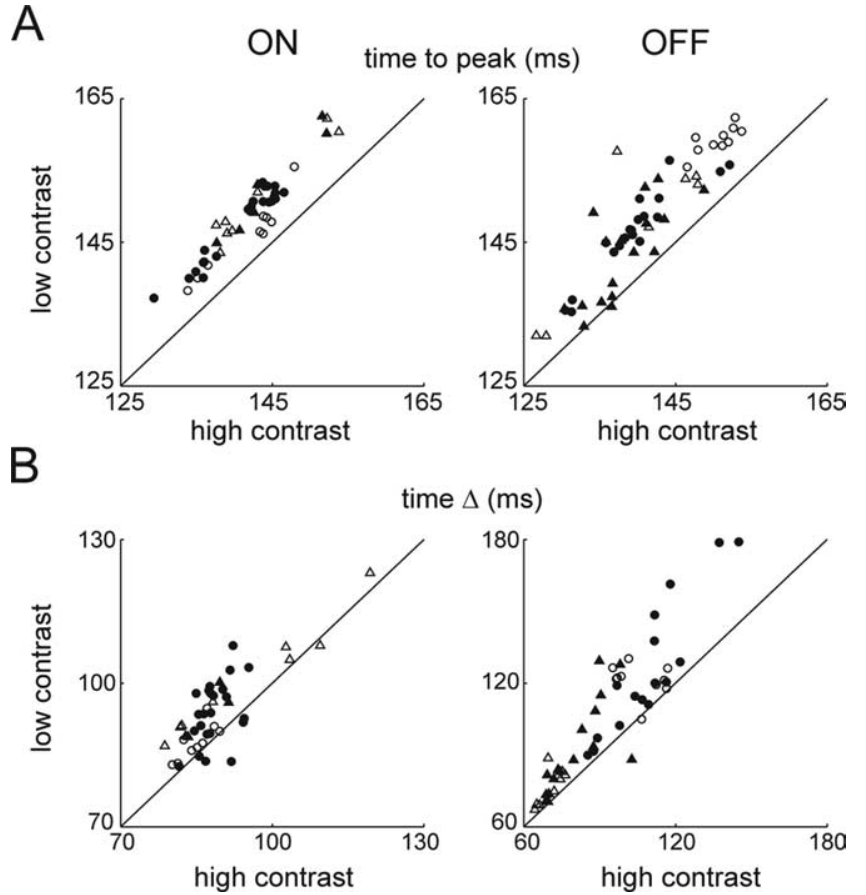

Figure 6. Changes in response kinetics during contrast adaptation. $\boldsymbol{A}, \boldsymbol{B}$, Time-to-peak and peak-to-trough interval for all recorded and classified ON (left) and OFF (right) RGCs $(n=100)$. $A$, Values during high contrast stimulation are plotted along the $x$-axis, and values during low contrast stimulation are along the $y$-axis. Throughout, open symbols signify cell recorded from control mice, and filled symbols mark results from dko mice. Circles indicate fast ON RGCs (left) and biphasic OFF cells (right). Triangles indicate medium ON RGCs (left) and monophasic OFF RGCS (right). B, For cells with biphasic filter functions (fast and medium ON RGCs and biphasic OFF RGCs) the time from peak-to-trough was measured under high ( $x$-axis) and low contrast ( $y$-axis) conditions. For monophasic OFF RGCs, this measure was substituted by determining the rise time from $5 \%$ to the peak of the STA. Symbols for the different cell types and genotypes are the same as in $\boldsymbol{A}$.

both $\mathrm{ON}$ and OFF pathways) compared with low contrast increments (signaled only by OFF pathways), in guinea pig Y cells was shown to be attributable to lower basal rates of glutamate release from OFF bipolar compared with ON bipolar cells and the fact that inhibition between pathways was unidirectional: ON to OFF (Zaghloul et al., 2003). Interestingly, several recent studies performed whole-cell recordings of mouse ON and OFF RGCs with large somata and dendritic field diameter $(180-350 \mu \mathrm{m})$ and found that OFF cells received prominent inhibition during $\mathrm{ON}$ phases of stimulation but not vice versa (Pang et al., 2003; Murphy and Rieke, 2006). It is tempting then to suggest that fast ON and biphasic OFF cells may belong to these large soma cells, because the size of spatial receptive fields we measured matches the dendritic field size reported from whole-cell recordings. Their large soma size could explain the preferred sampling in our multielectrode recordings, and preferential ON to OFF inhibition agrees with the different input/output relationships reported here. Given these and previously observed ON-OFF asymmetries, it is interesting to note that the medium ON cells showed higher threshold and similar gain compared with monophasic OFF cells. Thus, the response of medium ON cells at low effective contrast is as strongly rectified as the response of both types of OFF cells. This argues that asymmetries between ON and OFF pathways are likely to be cell type specific. Future experiments are needed to determine whether these variations result from differences in the balance of excitatory and inhibitory synaptic inputs, the mechanism of spike generation in RGCs, or a combination of
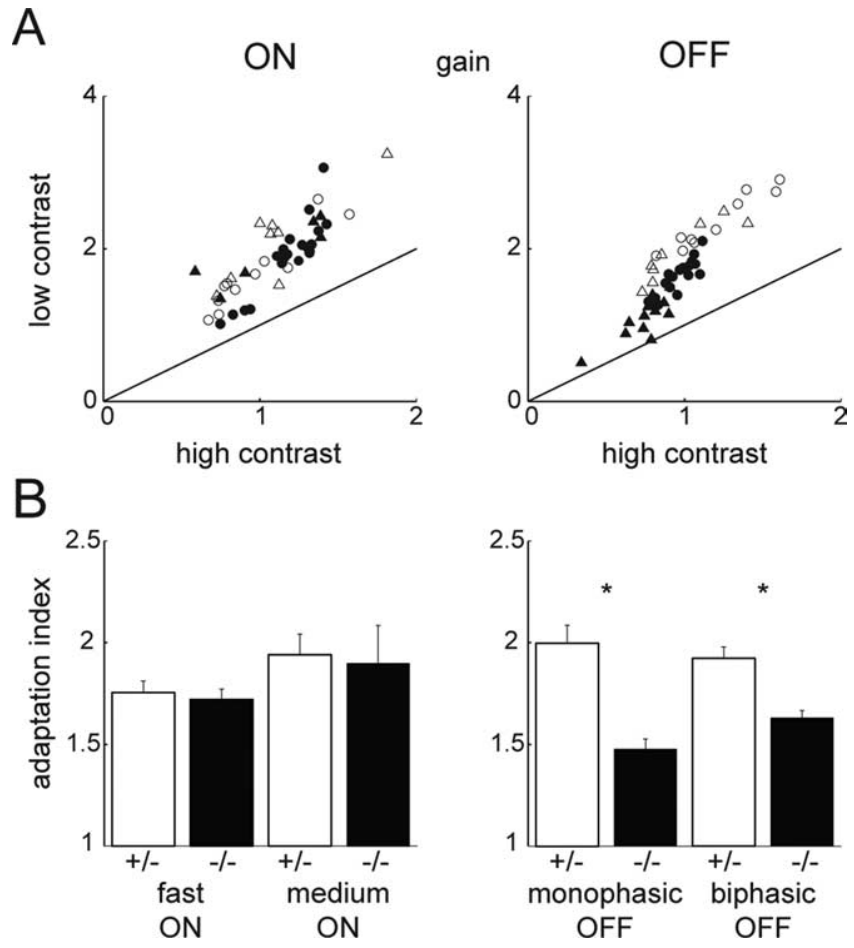

Figure 7. Contrast adaptation in response gain of RGCS. $A$, Gain of ON (left) and OFF (right) cells during the high contrast ( $x$-axis) or low contrast ( $y$-axis) stimulation. Gain is normalized by the median gain for all recorded and classified cells during high contrast stimulation. Open and filled symbols represent data from control and dko mice, respectively. Circles are used to mark values from fast ON RGCs (left) and biphasic OFF RGCs (right), and triangles are used to indicate data from medium ON (left) and monophasic OFF (right) RGCs. E, F, Adaptation index for the different cell types as indicated. Open and filled bars represent data from control $(+/-)$ and dko (-/-) mice, respectively. ${ }^{*} p<0.05$ using Wilcoxon-Mann-Whitney rank sum tests.

both (Margolis and Detwiler, 2007). In addition, how precisely RGC types identified in our study match up with those characterized in whole cell (Pang et al., 2003; Murphy and Rieke, 2006) and multielectrode recordings using square-wave modulated full-field stimulation (Carcieri et al., 2003) will require additional experiments.

Previous work showed overlapping defects in the expression of cell-specific markers in OFF but not ON cone bipolar cells of mice lacking either Vsx 1 or Irx5 (Chow et al., 2004; Ohtoshi et al., 2004; Cheng et al., 2005). In addition, OFF but not ON RGCs recorded from $V s x 1^{-1-}$ mice fired on average fewer action potentials in response to diffuse luminance steps compared with wild-type counterparts (Chow et al., 2004). Our results are in general agreement with these findings and extend them in several important ways. First, we show that defects in the expression profile of OFF bipolar cells are additive in dko mice compared with single gene mutants. In addition, bipolar cell number and gross morphology remain unchanged, making dko mice a suitable model for studying transcriptional control of circuit function. We found that space-time receptive fields of RGCs that determine spatial resolution and temporal filtering by retinal circuits are intact in both ON and OFF pathways of dko mice, and defects are limited to nonlinear aspects of circuit function. Interestingly these defects are cell type specific. Thus, the gain, response range, and threshold are lowered for biphasic OFF cells from dko compared with control mice, whereas only the threshold and gain are significantly lowered in monophasic OFF cells. Retinal circuits feature both such instantaneous as well as adaptive nonlinearities. We therefore analyzed adaptation to temporal 
contrast as an example of an adaptive nonlinearity to gain additional insight into the control of retinal circuit function by $V s x 1$ and $\operatorname{Ir} x 5$.

\section{Contrast adaptation in retinal circuits of control and dko mice}

The retinal circuitry, similar to other sensory systems (Brenner et al., 2000; Dean et al., 2005), adjusts its function to changes in input statistics to optimize encoding of the current stimulus environment with its limited output range. Accordingly, adaptations to both mean light levels and the extent of variations around the mean (i.e., contrast) have been documented in the retina of several species (for review, see Demb, 2002; Dunn et al., 2006; Wark et al., 2007). Our results on contrast adaptation in the response of mouse RGCs are in general agreement with previous studies of this process in other species. Thus, we found that, as in salamander, rabbit, guinea pig, and monkey, mouse RGCs shorten their integration time, reduce their gain, and raise their threshold in response to an increase in temporal contrast (Chander and Chichilnisky, 2001; Kim and Rieke, 2001; Baccus and Meister, 2002; Zaghloul et al., 2005). Also similar to previous observations in other species, the extent of contrast adaptation in mouse RGCs was cell type specific (Chander and Chichilnisky, 2001).

Contrast adaptation within the retinal circuitry is first observed in bipolar cells (Rieke, 2001; Baccus and Meister, 2002; Beaudoin et al., 2007), and, for both salamander and guinea pig, it was shown that adjustments in RGC responses are caused by a combination of adaptation in their bipolar cell input and adaptation in mechanisms of spike generation intrinsic to RGCs (Kim and Rieke, 2001; Zaghloul et al., 2005; Beaudoin et al., 2007). The importance of bipolar cells to contrast adaptation in the retina made it particularly interesting to assess this process in dko mice that show defects in the expression profiles of OFF cone bipolar cells. Indeed, we found that both types of OFF RGCs but not ON RGCs in dko mice showed greatly reduced adaptation to temporal contrast (Fig. 7). Strikingly, this effect was limited to adaptation of the response gain, and changes in the threshold and integration time were indistinguishable from control mice. This was surprising, because some previous models have considered adaptation in kinetics and gain inseparable (Victor, 1987). Instead, our results suggest that different mechanisms underlie these aspects of contrast adaptation in mouse RGCs.

We cannot exclude that part of the diminished contrast adaptation in OFF RGCs from dko mice is caused by changes intrinsic to RGCs. Nonetheless, the following observations argue against this. First, Vsx 1 and Irx 5 proteins appear not to be expressed in RGCs (Chow et al., 2001; Cheng et al., 2005). Moreover, if the lower firing rates observed for biphasic OFF cells and consequently reduced intrinsic spike frequency adaptation (Kim and Rieke, 2003) were to account for changes in contrast adaptation, it is hard to explain why these changes are identical in monophasic OFF cells that show the same peak firing rate in dko and control mice. For these reasons, we think that the reduced contrast adaptation observed in OFF RGCs in dko mice is likely to be, at least in part, a consequence of changes in their bipolar cell inputs.

How might these changes in contrast adaptation relate to changes in expression of marker proteins of OFF cone bipolar cells? Several of the proteins showing reduced expression in dko OFF cone bipolar cells are involved in $\mathrm{Ca}^{2+}$-homeostasis (CaBP5 and recoverin are cytosolic $\mathrm{Ca}^{2+}$-binding proteins, and PMCA1 is a plasma membrane $\mathrm{Ca}^{2+}$ pump), and contrast adaptation in salamander OFF cone bipolar cells has been shown to involve $\mathrm{Ca}^{2+}$-dependent mechanisms (Rieke, 2001). However, the number of proteins whose expression levels were tested here by immunohistochemistry form a small subset of those likely expressed in OFF bipolar cells. We therefore consider the reduced expression of the markers tested here and reduced contrast adaptation to have a common cause in changes in transcriptional control in dko mice but as yet cannot conclusively ascribe functional changes to specific proteins. To generate a more complete map of expression profiles in bipolar cells of dko mice and identify proteins that mediate the observed functional changes remains a challenge of future experiments.

Many aspects of the function of neural circuits, exemplified here in contrast adaptation in retinal signal processing, are of important value to the survival of the organism. It seems likely then that they evolved under specific control of genetic programs. In support of this notion, we find that the transcription factors $V s \times 1$ and Irx5 selectively control contrast adaptation in OFF pathways of the mouse retina. Interestingly, transcription factors are common among the immediate early genes that mediate changes in hippocampal circuit function during memory consolidation (Lanahan and Worley, 1998). Future experiments will have to show how prevalent such transcriptional controls of circuit level properties are across different networks in the central nervous system.

\section{References}

Baccus SA, Meister M (2002) Fast and slow contrast adaptation in retinal circuitry. Neuron 36:909-919.

Beaudoin DL, Borghuis BG, Demb JB (2007) Cellular basis for contrast gain control over the receptive field center of mammalian retinal ganglion cells. J Neurosci 27:2636-2645.

Bramblett DE, Pennesi ME, Wu SM, Tsai MJ (2004) The transcription factor Bhlhb4 is required for rod bipolar cell maturation. Neuron 43:779-793.

Brenner N, Bialek W, de Ruyter van Steveninck R (2000) Adaptive rescaling maximizes information transmission. Neuron 26:695-702.

Burmeister M, Novak J, Liang MY, Basu S, Ploder L, Hawes NL, Vidgen D, Hoover F, Goldman D, Kalnins VI, Roderick TH, Taylor BA, Hankin MH, McInnes RR (1996) Ocular retardation mouse caused by Chx10 homeobox null allele: impaired retinal progenitor proliferation and bipolar cell differentiation. Nat Genet 12:376-384.

Carcieri SM, Jacobs AL, Nirenberg S (2003) Classification of retinal ganglion cells: a statistical approach. J Neurophysiol 90:1704-1713.

Chander D, Chichilnisky EJ (2001) Adaptation to temporal contrast in primate and salamander retina. J Neurosci 21:9904-9916.

Cheng CW, Chow RL, Lebel M, Sakuma R, Cheung HO, Thanabalasingham V, Zhang X, Bruneau BG, Birch DG, Hui CC, McInnes RR, Cheng SH (2005) The Iroquois homeobox gene, Irx5, is required for retinal cone bipolar cell development. Dev Biol 287:48-60.

Chichilnisky EJ (2001) A simple white noise analysis of neuronal light responses. Network 12:199-213.

Chichilnisky EJ, Kalmar RS (2002) Functional asymmetries in ON and OFF ganglion cells of primate retina. J Neurosci 22:2737-2747.

Chow RL, Snow B, Novak J, Looser J, Freund C, Vidgen D, Ploder L, McInnes RR (2001) Vsx1, a rapidly evolving paired-like homeobox gene expressed in cone bipolar cells. Mech Dev 109:315-322.

Chow RL, Volgyi B, Szilard RK, Ng D, McKerlie C, Bloomfield SA, Birch DG, McInnes RR (2004) Control of late off-center cone bipolar cell differentiation and visual signaling by the homeobox gene Vsxl. Proc Natl Acad Sci USA 101:1754-1759.

Dean I, Harper NS, McAlpine D (2005) Neural population coding of sound level adapts to stimulus statistics. Nat Neurosci 8:1684-1689.

Demb JB (2002) Multiple mechanisms for contrast adaptation in the retina. Neuron 36:781-783.

Devries SH, Baylor DA (1997) Mosaic arrangement of ganglion cell receptive fields in rabbit retina. J Neurophysiol 78:2048-2060. 
Dunn FA, Doan T, Sampath AP, Rieke F (2006) Controlling the gain of rod-mediated signals in the mammalian retina. J Neurosci 26:3959-3970.

Feng L, Xie X, Joshi PS, Yang Z, Shibasaki K, Chow RL, Gan L (2006) Requirement for Bhlhb5 in the specification of amacrine and cone bipolar subtypes in mouse retina. Development 133:4815-4825.

Ghosh KK, Bujan S, Haverkamp S, Feigenspan A, Wassle H (2004) Types of bipolar cells in the mouse retina. J Comp Neurol 469:70-82.

Hatakeyama J, Tomita K, Inoue T, Kageyama R (2001) Roles of homeobox and bHLH genes in specification of a retinal cell type. Development 128:1313-1322.

Kim KJ, Rieke F (2001) Temporal contrast adaptation in the input and output signals of salamander retinal ganglion cells. J Neurosci 21:287-299.

Kim KJ, Rieke F (2003) Slow $\mathrm{Na}^{+}$inactivation and variance adaptation in salamander retinal ganglion cells. J Neurosci 23:1506-1516.

Lanahan A, Worley P (1998) Immediate-early genes and synaptic function. Neurobiol Learn Mem 70:37-43.

Levick WR (1967) Receptive fields and trigger features of ganglion cells in the visual streak of the rabbits retina. J Physiol (Lond) 188:285-307.

Livesey FJ, Cepko CL (2001) Vertebrate neural cell-fate determination: lessons from the retina. Nat Rev Neurosci 2:109-118.

Manookin MB, Demb JB (2006) Presynaptic mechanism for slow contrast adaptation in mammalian retinal ganglion cells. Neuron 50:453-464.

Margolis DJ, Detwiler PB (2007) Different mechanisms generate maintained activity in $\mathrm{ON}$ and $\mathrm{OFF}$ retinal ganglion cells. J Neurosci 27:5994-6005.

Murphy GJ, Rieke F (2006) Network variability limits stimulus-evoked spike timing precision in retinal ganglion cells. Neuron 52:511-524.

Ohtoshi A, Wang SW, Maeda H, Saszik SM, Frishman LJ, Klein WH, Behringer RR (2004) Regulation of retinal cone bipolar cell differentiation and photopic vision by the CVC homeobox gene Vsxl. Curr Biol 14:530-536.

Pang JJ, Gao F, Wu SM (2003) Light-evoked excitatory and inhibitory synaptic inputs to $\mathrm{ON}$ and OFF alpha ganglion cells in the mouse retina. J Neurosci 23:6063-6073.

Rieke F (2001) Temporal contrast adaptation in salamander bipolar cells. J Neurosci 21:9445-9454.

Sakai HM, Wang JL, Naka K (1995) Contrast gain control in the lower vertebrate retinas. J Gen Physiol 105:815-835.

Segev R, Puchalla J, Berry II MJ (2006) Functional organization of ganglion cells in the salamander retina. J Neurophysiol 95:2277-2292.

Smirnakis SM, Berry MJ, Warland DK, Bialek W, Meister M (1997) Adaptation of retinal processing to image contrast and spatial scale. Nature 386:69-73.

Victor JD (1987) The dynamics of the cat retinal X cell centre. J Physiol (Lond) 386:219-246.

Wark B, Lundstrom BN, Fairhall A (2007) Sensory adaptation. Curr Opin Neurobiol 17:423-429.

Wassle H (2004) Parallel processing in the mammalian retina. Nat Rev Neurosci 5:747-757.

Zaghloul KA, Boahen K, Demb JB (2003) Different circuits for ON and OFF retinal ganglion cells cause different contrast sensitivities. J Neurosci 23:2645-2654.

Zaghloul KA, Boahen K, Demb JB (2005) Contrast adaptation in subthreshold and spiking responses of mammalian Y-type retinal ganglion cells. J Neurosci 25:860-868.

Zeck GM, Xiao Q, Masland RH (2005) The spatial filtering properties of local edge detectors and brisk-sustained retinal ganglion cells. Eur J Neurosci 22:2016-2026. 